1. Van Breda Vriesman AC, Lohle PN, Coerkamp EG, Puylaert JB. Infarction of omentum and epiploic appendage: diagnosis, epidemiology and natural history. Eur Radiol 1999; 9: 1886-1892.

2. Legome EL, Belton AL, Murray RE, Rao PM, Novelline RA. Epiploic appendagitis: the emergency department presentation. J Emerg Med 2002; 22: 9.

3. Feber SS, Forman J. Appendices epiploicae: clinical and pathological considerations; report of 3 cases and statistical analysis on 105 cases. AMA Arch Surg 1953; 66: 329-338.

4. Son HJ, Lee SJ, Lee JH, Kim JS, Kim YH, et al. Clinical diagnosis of primary epiploic appendagitis: differentiation from acute diverticulitis. J Clin Gastroenterol 2002; 34: 435-438.

5. Rao PM, Rhea JT, Novelline RA, Mostafavi AA, McCabe CJ. Effect of computed tomography of the appendix on treatment of patients and use of hospital resources. N Engl J Med 1998; 338: 141.

6. Rioux M, Langis P. Primary epiploic appendagitis: clinical, US, and CT findings in 14 cases. Radiology 1994; 191: 523-526.

7. Singh AK, Gervais DA, Hahn PF, Sagar P, Mueller PR, et al. Acute epiploic appendagitis and its mimics. Radiographics 2005; 25: 1521-1534.

8. Sandrasegaran K, Maglinte DD, Rajesh A, Akisik FM. Primary epiploic appendagitis: CT diagnosis. Emerg Radiol 2004; 11: 9-14.

9. Puppala AR, Mustafa SG, Moorman RH, Howard CH. Small bowel obstruction due to disease of epiploic appendage. Am J Gastroenterol 1981; 75: 382 .

\section{Prevalencia de anemia en pacientes con insuficiencia cardiaca}

\section{Sr. Director:}

La anemia tiene una elevada prevalencia (15-30\%) en los pacientes con insuficiencia cardiaca $(1,2)$, incrementándose según la gravedad de la misma, del $9 \%$ para la clase I de la NYHA, hasta el $79 \%$ en la clase IV (3).

La insuficiencia cardiaca congestiva puede contribuir al desarrollo de la anemia y a su vez la anemia en el paciente que tiene una insuficiencia cardiaca constituye un factor de mal pronóstico, independiente para la mortalidad $(4,5)$.

La anemia de la insuficiencia cardiaca congestiva tiene un origen multifactorial y puede deberse a : a) déficit de hierro por disminución del aporte, malabsorción o pérdidas crónicas; b) disminución de la producción de eritropoyetina (EPO) por la insuficiencia renal crónica, que con elevada frecuencia presentan estos enfermos y también por la toma de fármacos de la familia de los inhibidores de la enzima de conversión de la angiotensina (IECA); c) pérdida urinaria de eritropoyetina y de transferrina por la proteinuria; d) inhibición de la eritropoyesis, por el factor de necrosis tumoral alfa que se encuentra elevado en estos pacientes, y e) hemodilucción, por el incremento del volumen plasmático (6).

Un déficit funcional de hierro, típico de la enfermedad crónica, puede desempeñar un papel importante en la anemia de los pacientes con insuficiencia cardiaca.

El tratamiento de la anemia en los pacientes con insuficiencia cardiaca, básicamente con hierro y EPO a dosis bajas, tiene una efectividad bien documentada (6).

Presentamos los resultados de un estudio en el que analizábamos la prevalencia de anemia en un grupo de pacientes diagnosticados de insuficiencia cardiaca. Excluimos del estudio los pacientes diagnosticados de insuficiencia renal crónica, hernia de hiato ó ulcus péptico gastroduodenal, procesos hematológicos o oncohematológicos, diversos cuadros reumatológicos crónicos que cursan frecuentemente con anemia y pacientes diagnosticados de anemia por cualquier otra causa.

También excluimos a los enfermos con enfermedad pulmonar obstructiva crónica (EPOC), dado que pueden presentar poliglobulia, que constituiría un factor de confusión en el estudio.

Realizamos un estudio retrospectivo, observacional y de prevalencia

Se incluyeron los enfermos ingresados por insuficiencia cardiaca como diagnóstico principal, en un servicio de Medicina Interna de un hospital del grupo 2, en el plazo de 9 meses, excluyendo los pacientes con los diagnósticos previos de exclusión referidos anteriormente.

Finalmente el grupo de estudio estuvo constituido por 46 pacientes, 26 mujeres y 20 varones con edades comprendidas entre 61 y 97 años, con una edad media de 78,8 años.

El valor medio de la hemoglobina fue de $12,9 \mathrm{~g} / \mathrm{dl}$.

Tenían anemia, definida como un valor de hemoglobina inferior a $12 \mathrm{~g} / \mathrm{dl}$, en las mujeres y de $13 \mathrm{~g} / \mathrm{dl}$ en los varones, un total de 14 pacientes $(30,4 \%)$.

Únicamente cuatro pacientes tenían un valor medio de hemoglobina inferior a 10 gramos/dl $(8,6 \%)$.

La edad media de estos 14 pacientes era de 75,8 años, por lo tanto ligeramente inferior a la global del grupo, pero sin diferencias relevantes.

No podemos precisar por no constar en el informe de alta en todos los casos, la clasificación de la gravedad de la insuficiencia cardiaca, aunque teniendo en cuenta la avanzada edad de los pacientes y los casos en los que estaba consignada (18 pacientes, 14 grado III y 4 grado IV), una gran mayoría de los mismos estaba encuadrado en los grupos III y IV de la NYHA.

Resulta llamativo la elevada proporción de pacientes con anemia que encontramos en nuestro estudio (30\%), aún más teniendo en cuenta el elevado número de pacientes que excluimos por presentar procesos que podrían justificar la anemia.

La mayoría de los pacientes con anemia fueron remitidos para estudio y seguimiento posterior en la consulta externa de Medicina Interna.

Como conclusiones podemos decir que la anemia es muy frecuente en los pacientes ancianos con insuficiencia cardiaca y aumenta con la gravedad de la enfermedad.

\section{F. Marcos Sánchez, M. I. Albo Castaño, D. Joya Seijo, P. del Valle Loarte, S. Casallo Blanco}

Servicio de Medicina Interna. Hospital Nuestra Señora del Prado. Talavera de la Reina. Toledo

1. Urrutia A, Lupón J, González B, Parajón T, Altimir S, Coll R, et al. Prevalencia de la anemia y su correlación con los parámetros clínicos en pacientes de una unidad multidisciplinaria de insuficiencia cardiaca. Med Clin (Barc) 2004; 122: 121-125.

2. Tanner H, Moschovitis GM, Hullin R, Pfiffner D, Hess OM, Mohacsi P. The prevalence of anemia in chronic heart failure. Int J Cardiol 2002; 86: 115-121.

3. Silverberg DS, Wesler D, Blum M, Keren G, Sheps D, Leibovitch E, et al. The use of subcutaneous erythropoietin and intravenous iron for the treatment of anemia of severe, resistant congestive heart failure improves cardiac and renal function and functional cardiac class, and markedly reduces hospitalisations. J Am Coll Cardiol 2000; 35: 1737-1744.

4. Ezekowitz JA, McAliste FA, Amstrong PW. Anemia is common in heart failure and is associated with poor outcomes. Circulation 2003; 107: 223225.

5. Sharma R, Francis DP, Pitt B, Poole-Wilson PA, Coats AJ, Anker SD. Haemoglobin predicts survival in patients with chronic heart failure: a substudy of the ELITE II trial. Eur Heart J 2004; 25: 1021-1028.

6. Silverberg DS, Wesler D, Blum M, Laina A. The importance of anemia and its correction in the management of severe congestive heart failure. Eur J Heart Failure 2002; 4: 681-686. 